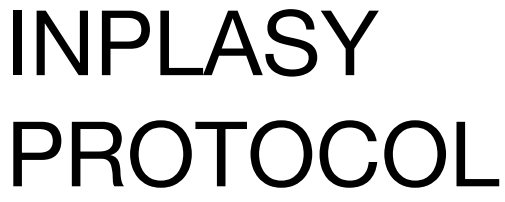

To cite: Geng et al. Efficacy and safety of acupuncture for Polycystic Ovary Syndrome undergoing in vitro

fertilization: A protocol for systematic review and metaanalysis. Inplasy protocol 202120069. doi:

10.37766/inplasy2021.2.0069

Received: 20 February 2021

Published: 21 February 2021

Corresponding author:

Huilan Du

duhuilan@163.com

Author Affiliation:

Hebei Key Laboratory of Integrative Medicine on LiverKidney Patterns, Institute of Integrative Medicine, College of Integrative Medicine, Hebei University of Chinese Medicine, Shijiazhuang, 050200, China

Support: None.

Review Stage at time of this submission: The review has not yet started.

Conflicts of interest:

None declared.

\section{Efficacy and safety of acupuncture for Polycystic Ovary Syndrome undergoing in vitro fertilization: A protocol for systematic review and meta-analysis}

Geng, JR¹; Lv, JM²; Zhang, SC³ Ma, YC4; Liang, X5; Sun, Y4; $\mathrm{Du}, \mathrm{HL}$.

Review question / Objective: Acupuncture has been used in the treatment of PCOS infertility and widely applied in the field of assisted reproduction. But its efficacy has not been evaluated scientifically and systematically. The aim of this meta-analysis of randomized controlled trials is to provide a systematic evaluation to assess the efficacy and safety of acupuncture in patients with polycystic ovary syndrome undergoing in vitro fertilization.

Condition being studied: Polycystic ovary syndrome (PCOS) is one of the most common endocrine disorders in women of reproductive age, which can cause ovulation disorder infertility. In vitro fertilization (IVF) as a method to treat ovulation disorder infertility in polycystic ovary syndrome, however, there are still low clinical pregnancy rate, high complications and other problems. Thus, it is necessary to explore new methods to improve the success rate.Acupuncture as a treatment for infertility has been used for thousands of years. In recent years, acupuncture has been widely used in the treatment of polycystic ovary syndrome and in vitro fertilization, while the curative effect of acupuncture is controversial. Thus, we will comprehensively collect evidence, extract and analyze data, draw reasonable and objective conclusions, in order to provide better guidance to clinicians in the treatment of PCOS undergoing in vitro fertilization.

INPLASY registration number: This protocol was registered with the International Platform of Registered Systematic Review and Meta-Analysis Protocols (INPLASY) on 21 February 2021 and was last updated on 21 February 2021 (registration number INPLASY202120069).

\section{INTRODUCTION}

Review question / Objective: Acupuncture has been used in the treatment of PCOS infertility and widely applied in the field of assisted reproduction. But its efficacy has not been evaluated scientifically and systematically. The aim of this meta- 
analysis of randomized controlled trials is to provide a systematic evaluation to assess the efficacy and safety of acupuncture in patients with polycystic ovary syndrome undergoing in vitro fertilization.

Condition being studied: Polycystic ovary syndrome (PCOS) is one of the most common endocrine disorders in women of reproductive age, which can cause ovulation disorder infertility. In vitro fertilization (IVF) as a method to treat ovulation disorder infertility in polycystic ovary syndrome, however, there are still low clinical pregnancy rate, high complications and other problems. Thus, it is necessary to explore new methods to improve the success rate.Acupuncture as a treatment for infertility has been used for thousands of years. In recent years, acupuncture has been widely used in the treatment of polycystic ovary syndrome and in vitro fertilization, while the curative effect of acupuncture is controversial. Thus, we will comprehensively collect evidence, extract and analyze data, draw reasonable and objective conclusions, in order to provide better guidance to clinicians in the treatment of PCOS undergoing in vitro fertilization.

\section{METHODS}

Participant or population: The study will include women diagnosed with PCOS and receiving IVF, regardless of race, educational level, and source of cases.

Intervention: The experimental group should be treated with acupuncture (including manual acupuncture and electroacupuncture).

Comparator: The control group should be treated with no acupuncture or sham acupuncture.

Study designs to be included: All clinical randomized controlled trials (RCTs) will be included in this review.
Eligibility criteria: We will only include randomized controlled trials (RCTs) of acupuncture for PCOS undergoing IVF, regardless of reviews, protocols, animal experiments, case studies, non-therapeutic clinical studies.

Information sources: This study will search the following database: EMBASE, PubMed, Web of Science, the Cochrane Central Register of Controlled Trials (CENTRAL), and four Chinese databases: VIP, Wanfang, CNKI, and the Chinese Biomedical Literature Database (CBM).

Main outcome(s): The main outcomes are the clinical pregnancy rate (CPR), the live birth rate (LBR), the incidence of ovarian hyper-stimulation syndrome (OHSS),dose of $\mathrm{Gn}$, duration of $\mathrm{Gn}$ stimulation.

Additional outcome(s): The additional outcomes involved the biochemical pregnancy rate (BPR), the ongoing pregnancy rate (OPR), serum hormone level, the cycle cancellation rates, and adverse events (AEs).

Quality assessment / Risk of bias analysis: Two researchers will evaluate the quality of RCTs by using the risk assessment tool recommended in Cochrane Handbook 5.1.0. The following domains will be accessed: generation of random sequences, blinding of investigators and participants, blinding of study results, completeness of outcome data, selectivity in reporting of results, and other biases. If the data reported in the document is insufficient, we will contact the author of the experiment for consultation and resolution. However, if we fail to contact the author, we will analyze only the data that are useful in the literature or analyze the missing data in the discussion.

Strategy of data synthesis: The data will be entered into the Review Manager software (RevMan 5.3).After the data extraction, the reviewers will determine if there is a possibility of performing a meta-analysis, by considering if the heterogeneity is moderate or strong, as assessed by 12 $(25 \%-50 \%$, moderate heterogeneity and $>$ 
$50 \%$ strong heterogeneity), the random effects model will be used for analysis. This study involves bicategorical and continuous variables. We will express the bicategorical data as the relative risk(RR) with $95 \%$ confidence interval $(\mathrm{Cl})$. And for the continuous data, we will express the results as the difference or standard mean difference (SWD) with $95 \% \mathrm{CI}$.

Subgroup analysis: If $12 \geqq 50 \%$, substantial heterogeneity is considered to be present. If heterogeneity is present, we will analyze the cause through subgroup analysis.We will establish subgroup according to the different comparisons, or other factors that may affect outcomes, such as age, interval and duration of treatment, etc.

Sensitivity analysis: To ensure the stability and reliability of the results, a sensitivity analysis will be performed. The main contents of the analysis include the impact of method quality, sample size, and missing data on the study. Excluding the studies which were poor quality or potential contributors to heterogeneity, the metaanalysis will be reused. We will compare the results and discuss.

Language: No restriction.

Country(ies) involved: China.

Keywords: polycystic ovarian syndrome; acupuncture; in vitro fertilization; metaanalysis; protocol.

Contributions of each author:

Author 1 - Jingran Geng.

Author 2 - Jinmeng Lv.

Author 3 - Shuancheng Zhang.

Author 4 - Yucong Ma.

Author 5 - Xiao Liang.

Author 6 - Ying Sun.

Author 7 - Huilan Du. 